\title{
A Note on Morato on Modality and Explanation
}

\author{
Nathan Wildman ${ }^{1}$
}

Received: 22 December 2015/ Accepted: 4 November 2016/Published online: 8 February 2017

(C) The Author(s) 2017. This article is published with open access at Springerlink.com

\begin{abstract}
This brief note critically assesses the central arguments in Morato's (Erkenntnis 79:327-349, 2014) recent contribution to the growing literature on Blackburn's dilemma about necessity. In particular, I demonstrate that (i) neither of Morato's two novel reconstructions of the dilemma's contingency horn succeed, since both turn on false premises; and, (ii) Morato fails to adequately motivate his own response to these reconstructions. The upshot is that Morato has set himself a pair of flawed problems, then offered a flawed solution.
\end{abstract}

Blackburn (1986) offers a dilemma for any realist attempt to explain the source of necessity. In brief: suppose we explain $p$ 's necessity by appeal to some $q$. Since explanation is factive, $q$ must be true. And, if true, it must itself be either necessary or contingent. The problem is that either option leads to trouble: if $q$ is necessary, there is a 'bad residual must' concerning $q$ 's own modal status, and if $q$ is contingent, there is 'strong pressure to feel that the original necessity has not been explained or identified, so much as undermined.' Consequently, either the explanans 'shares the modal status of the original, and leaves us dissatisfied, or it does not, and leaves us equally dissatisfied' (Blackburn 1986: 54).

Blackburn intended for this dilemma to motivate a wholesale abandoning of realism in favour of quasi-realism about modality. Few have been so moved. This is partially because Blackburn does little more than provide the above sketch of the dilemma's two horns. As a result, a cottage industry has developed around

Nathan Wildman

nathan.wildman@uni-hamburg.de

1 Department of Philosophy, Universität Hamburg, Von-Melle-Park 6, 20146 Hamburg, Germany 
constructing - or, more charitably, reconstructing — the arguments underpinning the two horns. ${ }^{1}$

Recently, Morato (2014) has offered two novel reconstructions of the dilemma's contingency horn, along with a diagnosis of how he thinks would-be contingentists should best respond to them. In the process, Morato makes a case for a fairly radical 'trans-world' view about the nature of explanation in modal contexts.

This brief note critically assesses Morato's contribution to the debate about Blackburn's dilemma. In particular, I demonstrate that (i) neither of Morato's two novel reconstructions succeed, since both turn on false premises (though not the ones that Morato himself suggests); and, (ii) Morato's own response to them, which involves rejecting the intra-worldly factivity of explanation, is unmotivated at best. The general upshot is that while Morato is no threat to contingentists, he is not much help either.

\section{Morato's two arguments and his response thereto}

Morato's initial reconstruction of Blackburn's contingency horn features two central assumptions. The first is:

(Ex-Nec) ' $\square p$ because $q$ ' is true in a world $w_{i}$ iff, for every $w, p$ because $q$ in Morato claims that (Ex-Nec) follows from the standard, 'worldly' definition of necessary truth-that ' $\square p$ ' is true in world $w$ iff for every $w, p$ in $w$-and a straightforward 'disquotation' principle:

(Ex-Rel) ' $p$ because $q$ ' is true in world $w$ iff ( $p$ because $q$ ) in $w$

Meanwhile, the second key assumption, which falls out of the factivity of explanation, is:

(Truth-Ans) For all worlds $w,((p$ because $q)$, in $w \rightarrow q$ in $w)$

With these in place, Morato offers the following argument. Assume, for reductio, that $\square p$ because $q$, where $q$ is only contingently true. As $q$ is contingent, there is a world $w_{i}$ where $q$ is false. Given that $p$ is necessary, $p$ is true in $w_{i}$. By (Truth-Ans), it follows that it is not the case that $p$ because $q$ in $w_{i}$. So, by (Ex-Nec), it follows that, contra the initial assumption, it is not the case that $\square p$ because $q$. Thus, given $(\mathrm{Ex}-\mathrm{Nec})$ and (Truth-Ans), contingent necessity-makers are impossible. ${ }^{3}$

\footnotetext{
${ }^{1}$ See e.g. Hale (2002, 2013), Hanks (2008), Lange (2008), and Cameron (2010).

2 This and the following two principles are slightly modified from Morato (2014: 336). I have replaced all instances of ' $q$ explains p', with ' $p$ because q' and flipped the ' $p$ 's and ' $q$ 's to better correspond to the order of presentation. Neither alteration changes anything of substance. Per standard, I assume that 'because'-like the notion of explanation it is meant to express-is factive, irreflexive, antisymmetric, and transitive.

${ }^{3}$ Morato credits Cameron (2010) with a similar argument, though Cameron does not use anything like $(\mathrm{Ex}-\mathrm{Nec})$.
} 
Morato's second reconstruction starts with the thought that (Ex-Rel) is compatible with contingent explanatory relations-i.e., with ' $p$ because $q$ ' being true in some world $w_{1}$, but false in $w_{2}$. Since this might be 'objectionable' to those who think explanations have some 'modal force' (2014: 337), he suggests a replacement:

(Ex-Rel-Strong) ' $p$ because $q$ ' is true in world $w_{i}$ iff $p$ because $q$ in $w_{i}$ and, for every $w$ where $p$ is true, $p$ because $q$

Employing this principle, Morato offers his second reconstruction:

Assume that a contingent proposition, $q$, explains $\square p$. [By (Ex-Rel-Strong)], if $q$ explains $\square p$, then $q$ explains $\square p$ in every possible world in which $\square p$ is true. Given that $\square p$ is true in every possible world, then [by (Truth-Ans)] $q$ is true in every possible world, therefore $q$ is necessary, contrary to our assumption. (2014: 338)

Having set these two reconstructions up, Morato then proceeds to knock them down. Specifically, he contends that both turn on a false premise: (Truth-Ans). According to Morato, as it requires that $q$ must be true in world $w$ to explain something in $w$, (Truth-Ans) entails that '(mere) possibilities cannot explain anything'. Consequently, (Truth-Ans) is incompatible with cases where possibilities 'occupy the role of explanantia' (2014: 340).

Building on this, Morato claims that such cases appear 'in a great number of philosophical contexts', though he mentions only two specific instances: (i) modal arguments, where an actual truth (e.g. the non-identity of a statue and lump of clay) is explained by a non-actual possibility (e.g. the lump survives being smashed but the statue doesn't); and (ii) discussions of moral responsibility, where the actual moral responsibility of an agent is explained in terms of 'non-actual courses of actions available to him at the moment of [a] choice' (2014: 341-342).

Moreover, Morato notes that we occasionally explain the possibility of something even when it is in fact false, which we do by showing that the relevant claim is compatible with what is actually true. So, to explain

how it would have been possible for [Morato] to be elected in Parliament in the last general elections, what I need to do is explain how [his] actual properties before the last general election were not incompatible with [his] possible becoming a member of Parliament. (2014: 341)

Consequently, there is a proposition $-<$ Morato is elected $>$ - whose possible truth we explain by appealing to facts about metaphysical compatibility. This, says Morato, constitutes a counter-example to

(Truth-Dum) $\quad\left(p\right.$ because $q$ is true in $\left.w_{i}\right) \rightarrow\left(p\right.$ is true in $\left.w_{i}\right)$

But there is a symmetry within the explanans and explanandum roles, which also makes this a counter-example to (Truth-Ans). For suppose that (a) $p$ in $w_{1}$ because $q$ in $w_{2}$, (b) $\neg q$ in $w_{1}$, and (c) $\neg p$ in $w_{2}$. Relative to $w_{2}$, the explanation in (a) is an instance of an actual truth, $q$, explaining a (mere) possibility, $p$. However, relative to 
$w_{1}$, (a) is an instance of an actual truth being explained by a (mere) possibility. Thus it amounts to a counter-example to (Truth-Ans).

For these reasons, Morato rejects (Truth-Ans). And, as (Truth-Ans) plays a role in both, he also rejects his two reconstructions of Blackburn's contingency horn.

\section{On Morato's case against (Truth-Ans)}

Before responding to Morato's case against (Truth-Ans), it is worth noting that the principle is only incompatible with true explanatory claims where the explanans is false at the world of assessment - that is, (Truth-Ans) is false iff ' $((p$ because $q)$ and $\neg q$ )' is true at some world $w$. In this way, (Truth-Ans) is perfectly compatible with possibilities serving as explanantia-for example, the truth of 'I am not necessarily an only child because I possibly have a sister' is compatible with (Truth-Ans), since the explanans is actually true. What is incompatible are true explanations involving a mere possibility, where $q$ is a mere possibility in $w$ iff $q$ is false, though possibly true, in $w$.

This highlights that rejecting (Truth-Ans) amounts to denying that explanation is intra-worldly factive - that is, denying that ' $p$ because $q$ ' is true in $w$ only if both ' $p$ ' and ' $q$ ' are true in $w$. As it is extremely plausible, abandoning intra-world factivity isn't a move to be taken lightly. Hence the burden of proof is on Morato to motivate this radical step. However, nothing Morato has offered does so; or, put more generally, Morato has not provided any genuine counter-examples to (Truth-Ans).

Take the modal argument case. Here, the explanans is not a false (but possibly true) claim, but a true possibility claim - the relevant explanation is not, 'Statue and Lump are distinct because Statue persists and Lump does not'; rather, it is, 'Statue and Lump are distinct because possibly, Statue persists and Lump doesn't'.

Similarly, the explanation in the moral responsibility case is something like, 'A is morally responsible for her actions because possibly, A behaves otherwise'. Again, the explanans is not the false (but possibly true), 'A behaves otherwise', but the true possibility claim, 'Possibly, A behaves otherwise'. 4

In other words, both cases are explanations of the form ' $(p$ because $\diamond q)$ in $w$ ', not ' $\left(p\right.$ in $\left.w_{i}\right)$ because $\left(q\right.$ in $\left.w_{i i}\right)$ '. And given that the explanans is true in both, neither tells against (Truth-Ans) since a counter-example to the principle requires a false explanans.

Morato might respond that the distinction between ' $(p$ because $\diamond q)$ in $w$ ' and ' $\left(p\right.$ in $\left.w_{i}\right)$ because $\left(q\right.$ in $\left.w_{i i}\right)$ ' collapses, because ' $\diamond p$ and $p$ have the same content, [and] therefore should be credited with the same explanatory potential' (2014: 345). Consequently, the explanans is false in the world of assessment, and the cases are counter-examples to (Truth-Ans) after all.

I must confess that I have some difficulty wrapping my head around the idea that $\diamond p$ and $p$ have the same content, as they frequently differ in truth value, and certainly differ in entailments. But, setting this aside, it is clear that there is an explanatory difference between possibility claims and their non-modalized

\footnotetext{
${ }^{4}$ In effect, the objection here is a version of Morato's 'Third Problem' (2014: 345).
} 
correlates, for there are cases where $p$ is (at least) a partial explanation for $\diamond p$, though $\diamond p$ is never a partial explanation for itself. For example, " $\diamond(O h l e$ is a dog) partially because Ohle is a dog' is actually true, though ' $\diamond($ Ohle is a dog) partially because $\diamond($ Ohle is a dog $)$ ' is necessarily false. This difference in explanatory potential gives us good reason to think there is a distinction between ' $p$ because $\diamond q)$ in $w$ ' and ' $\left(p\right.$ in $\left.w_{i}\right)$ because $\left(q\right.$ in $\left.w_{i i}\right)$ '.

Alternatively, Morato might respond by limiting the kind of propositions that can enter into explanation. Early in his article, Morato stipulates that, 'for simplicity's sake' (2014: 328fn1), his principles are all restricted to propositional atoms. Obviously, such a restriction would block the above analysis: no possibility claim could be an explanans (or explanandum), so the only possible reading of the modal argument and moral responsibility cases would involve a false atomic explanans.

But this indicates that Morato's restriction does more than merely reduce complexity - in fact, this stipulation does most of the heavy lifting, making the principles look more plausible than they actually are. So, why, other than simplification, ought we restrict ourselves to just propositional atoms?

Morato claims that limiting the principles to propositional atoms is "motivated by the assumption that to explain a modal formula is to explain the corresponding nonmodal formula in the possible worlds quantified over by the modal operator' (2014: 340fn15), which he formalizes as

' $\diamond p$ because $q$ ' is true in $w$ iff for some world $w, p$ because $q$ in $w$

However, as the right-hand-side states the truth conditions for ' $\diamond(p$ because $q)$ ', this entails

' $\diamond p$ because $q$ ' is true in $w$ iff $\diamond(p$ because $q)$

Yet this is false: generally, an explanation for something's modal status need not be the same as a (possible) explanation of that thing. For example, many think that while the truth of 'Socrates is wise' explains the truth of '(Socrates is wise or $\neg$ (Socrates is wise) )', the former does not explain the latter's modal status. ${ }^{5}$ And, more to the point, while 'Ohle is a dog because Ohle is a dog' is necessarily false, ' $\left(\diamond\right.$ Ohle is a dog) because Ohle is a dog' is plausibly true. ${ }^{6}$ Yet Morato's restriction rules this explanation out. This, in combination with the above, means there is little reason to accept the restriction. And once the restriction goes, the two initial cases against (Truth-Ans) look inconclusive at best.

A similar point applies to Morato's second argument against (Truth-Ans). He is correct that, if we have a scenario where (i) ' $p$ because $q$ ' is true, (ii) ' $p$ ' is false, and (iii) ' $\diamond p$ ' is true, then both (Truth-Dum) and (Truth-Ans) would be false. But, in the case he details, condition (ii) is not met: what is explained is not a false non-modal claim but a true possibility claim. Or, to put the point another way, the explanation

\footnotetext{
${ }^{5}$ If it did, we would have a straight case of a contingency explaining the modal status of a necessity, and hence a counter-example to Blackburn's contingency horn regardless of formulation. See Wildman (ms) for further discussion.

${ }^{6}$ Further, Morato's restriction rules out natural explanations of iterated modalities via appeal to simpler modal formula—for example, ' $\diamond \diamond p$ because $\diamond$ ' and ' $\square \square p$ because $\square p$ ' are, assuming the restriction, illegitimate explanations.
} 
is of the form ' $(\diamond p$ because $q)$ in $w$ ', and the explanandum is the (true!), ' $\diamond$ (Morato was elected)'. This fits with Morato's 'symmetry' point-depending on which world you're evaluating from, either the explanans or the explanandum will be a (true!) possibility-but leaves (Truth-Dum) and (Truth-Ans) unharmed. So Morato's second point against (Truth-Ans) is also inconclusive.

These failures undercut Morato's suggested abandoning of (Truth-Ans). But, again, this is not problematic, since (Truth-Ans) does not, pace Morato, entail that possibilities cannot play a role in explanations. All it entails is that there are no worlds where ' $((p$ because $q)$ and $\neg q)$ ' is true-and given the plausibility of the intra-worldly factivity of explanation, this is a happy result.

\section{On Morato's two reconstructions}

Of course, Morato rejected (Truth-Ans) in order to block his two reconstructions of Blackburn's contingency horn. So contingentists might worry that, by embracing (Truth-Ans), they have jumped from the frying pan and into fire. Thankfully, they need not despair-as it happens, there are other reasons for rejecting the two.

The weak point of the first reconstruction is $(\mathrm{Ex}-\mathrm{Nec})$. This principle is unmotivated: what follows from the standard worldly definition of ' $\square$ ' and (Ex-Rel) is not $(\mathrm{Ex}-\mathrm{Nec})$, but rather

(Ex-Weak) ' $\square p$ because $q$ ' is true in world $w_{i}$ iff (i) for all $w, p$ in $w$; (ii) $q$ in $w_{i}$; and, (iii) ( $\square p$ because $q$ ) in $w_{i}$

And plugging (Ex-Weak) into the first argument renders it harmless to contingentists.

Moreover, (Ex-Nec) is false. In general, any time $q$ is either identical to $p$ or is a conjunction that has $p$ as a conjunct, then (Ex-Nec)'s right-hand-side is, due to the irreflexivity of explanation, necessarily false. So, for example, ' $\square(2+2=4)$ because $(\square(2+2=4)$ and $\forall x(\square x \rightarrow \square \square x))$ ', is necessarily false. However, it is natural to think that ' $\square \square(2+2=4)$ because $(\square(2+2=4)$ and $\forall \mathrm{x}(\square \mathrm{x} \rightarrow \square \square \mathrm{x}))^{\prime}$ is actually true - that is, the necessity plus the generalization explains the necessity's being necessarily necessary. ${ }^{7}$ But this falsifies (Ex-Nec): the right-hand-side is true, the left-hand-side false. ${ }^{8}$ Consequently, the first reconstruction is no threat to contingentists; it features a premise that everyone should reject.

A similar point applies to Morato's second reconstruction: a central premise(Ex-Rel-Strong) - is problematic. First, this principle is obviously false when we consider cases of explanation overdetermination. For example, true existential generalizations are, per standard, explained by their true instances. Thus 'there is a dog because Ohle is a dog' is actually true. However, as Ohle only contingently exists, there is a world $w_{1}$ where the explanans is false. But there might be other

\footnotetext{
7 The point remains if we shift from a straight generalization to a law.

${ }^{8}$ Morato might reply by appealing to his stipulation that his principles are restricted to logical atoms, but, as argued earlier, this restriction is problematic.
} 
dogs in $w_{1}$, whose existence serves to explain the existential. Thus there are cases where the relevant $p$ is true though both $q$ and ' $p$ because $q$ ' are false.

In fact, we need not restrict ourselves to overdetermination cases for counterexamples. If we assume $p$ only contingently obtains, then ' $(p$ or $\neg p)$ because $p$ ' might be true in world $w_{1}$, though there will be a world $w_{2}$ where ' $(p$ or $\neg p$ ) because $p$ ' is false and, instead, ' $(p$ or $\neg p)$ because $\neg p$ ' is true.

Moreover, (Ex-Rel-Strong) does not capture the idea that explanatory relations have modal force. ${ }^{10}$ Rather, a plausible principle that does is:

(EMF) ' $p$ because $q$ ' is true in world $w_{i}$ iff

(i) $\quad\left((p\right.$ because $q)$ in $\left.w_{i}\right)$; and

(ii) for every $w$ where ' $q$ ' and ' $p$ ' are true, ( $p$ because $q$ ) in $w$

However, (EMF) neuters Morato's second reconstruction-the closest we get to a contradiction is that every world where both $\square p$ and $q$ are true is a world where ' $\square p$ because $q$ ' is true, which is perfectly compatible with $q$ 's being contingent.

The upshot is that neither of Morato's reconstructions are problematic: both feature a false premise, the sensible replacement for which leaves the argument impotent.

\section{Conclusions}

In summary, both of Morato's novel reconstructions rely upon false premises (though not the ones that Morato suspected), and therefore are not a threat to those who would go in for contingent necessity-makers. Meanwhile, Morato's case for rejecting (Truth-Ans) is less than convincing, especially once we are clear that doing so amounts to denying the intra-worldly factivity of explanation.

Of course, I have said nothing here against Morato's 'trans-world' notion of explanation, which suggests a new and interesting way to think about the relationship between explanation and modality. Similarly, Morato's challenge to clarify the explanatory difference between modalized and non-modalized claims is a difficult and worthwhile one. However, these points do not take away from the fact that, when it comes to the contingency horn of Blackburn's dilemma, it seems that Morato set himself a pair of flawed problems, then offered a flawed solution.

\footnotetext{
9 Thanks to an anonymous referee for this example. In fact, I have elsewhere argued that cases might involve contingent necessity-makers; see Wildman (ms).

10 To his credit, Morato (2014: 339) also argues that (Ex-Rel-Strong) is dubious, though he does not offer the above (decisive) counter-examples.
} 
Open Access This article is distributed under the terms of the Creative Commons Attribution 4.0 International License (http://creativecommons.org/licenses/by/4.0/), which permits unrestricted use, distribution, and reproduction in any medium, provided you give appropriate credit to the original author(s) and the source, provide a link to the Creative Commons license, and indicate if changes were made.

\section{References}

Blackburn, S. (1986). Morals and Modals. In G. Mcdonald \& C. Wright (Eds.), Fact, Science, and Morality: Essays on A.J. Ayer's Language, Truth, and Logic (pp. 119-141). Oxford: Blackwell. (Reprinted in Blackburn, S. (1993). Essays in quasi-realism, pp. 52-74, Oxford: Oxford University Press.).

Cameron, R. (2010). On the source of necessity. In B. Hale \& A. Hoffman (Eds.), Modality (pp. 137-153). Oxford: Oxford University Press.

Hale, B. (2002). The source of necessity. Noûs, 36, 299-319.

Hale, B. (2013). Necessary beings: An essay on ontology, modality, and the relations between them. Oxford: Oxford University Press.

Hanks, P. (2008). A dilemma about necessity. Erkenntnis, 68(1), 129-148.

Lange, M. (2008). Why contingent facts cannot necessities make. Analysis, 68(298), 120-128.

Morato, V. (2014). Explanation and modality: On the contingency horn of Blackburn's dilemma. Erkenntnis, 79, 327-349.

Wildman, N. (ms.). Necessity by accident. Unpublished manuscript. 